\title{
Virtual Gaming Environments as our Future Adobe
}

\author{
Kang-Hyuk Lee \\ Department of Game Engineering \\ Tongmyong University, Busan, 608-711, Korea
}

\begin{abstract}
Currently, the argument that humanity will eventually reside in some sort of a simulated environment with unlimited resources is actively being discussed in the realm of science and engineering. This paper addresses the issue from the perspective of computer engineering, more specifically in terms of the future gaming environment which is very likely to be brought to us in the form of virtual reality probably in the not-so-distant future. In so doing, Bostrom's simulation argument[1] and Kurzweil's singularity[5] are reviewed, and how our future adobe indistinguishable from our 'real' reality that may be attained by explosive technological advancement relates to the future gaming environment. The problem of human consciousness which is inevitably intertwined with the issue of living in a virtual reality environment is also addressed.
\end{abstract}

Keywords: Computer Simulation, Virtual Reality, Computer Game, Consciousness

\section{INTRODUCTION}

In the Feb. 21st edition of TIME magazine, its cover story, provocatively titled "2045: The Year Man Becomes Immortal”, charged at the issue of immortality, though the illustration for the story seems to take on too much of Matrix-like punchingin-the-back-of-the-neck brain-connecting device. Mainly focusing on Ray Kurzweil's work on 'Singularity' explicated in his mind-boggling book, "The Singularity is near" [5], the story packs people in the theater of immortality, which our technological advancement may embody in the future system, whether it is mechanical, biochemical, or purely softwareengineered.

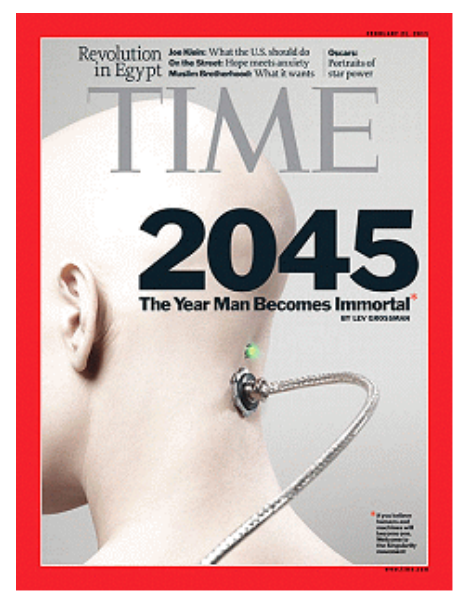

Fig. 1. The illustration of TIME's cover story

\footnotetext{
*Corresponding author.E-mail : klee@tu.ac.kr Manuscript received Sep 30, 2011 ; accepted Dec.13, 2011
}

In this paper, we address the issue of living in a virtual reality, probably forever, from the perspective of computer engineering. More specifically, I deal with the issue in relation with the future gaming environment which is very likely to be brought to us in the form of virtual reality in the not-so-distant future. The organization of this paper is as follows. In section 2, the possible future environments that two important futurists believe we will end up living in will be discussed. In so doing, Bostrom's Simulation Argument and Kurzweil's Singularity are introduced and reviewed. In section 3, these future environments will also be discussed in the context of future gaming environment. Section 4 addresses the issue of human consciousness in relation with technological environments discussed in section 2 and 3 . The section 5 summarized the paper.

\section{VIRTUAL REALITY: ANY WAY YOU WANT IT}

As Llinás suggests in his famous book titled "I of the Vortex"[7], our reality also is 'virtual' in the sense that we do not see the world as it is, but do see the world reflected in the retina that is connected to our brain by the optic nerve. We already witnessed a variety of virtual realities (VR) ${ }^{1}$ as our ultimate future adobe in many Sci-Fi novels and movies. However, it has also been addressed in depth by many renowned academics from various fields. It doesn't seem

\footnotetext{
1 Throughout the paper, VR will be used as the simulated environment that is technologically actualized and indistinguishable from our 'real' reality. The term "computer simulation" will also be interchangeably used with VR.
} 
weird-sounding any more that VR (or computer simulation) will be the future environment that humanity will live in, whatever the form of life they would take on. In this section, I will review the possible future environment that some futurists believe we will end up living in. It will also be discussed in the context of future gaming environment.

\subsection{Bostrom's Simulation Argument}

Since its publication[1], Bostrom's so-called "Simulation Argument" has attracted much attention from academics in a wide range of disciplines and interested laymen alike. The popularity of his argument lies, as one of his tripartite disjoints strongly suggests, in a logical possibility that we may be already living in some sort of computer simulation. ${ }^{2} \mathrm{He}$ persuasively estimates the human brain process in terms of computation, citing various works on this issue including his own and how it can be dealt with by utilizing powerful computers which must be available in the future however distant it is. ${ }^{3}$

These future computers will be powerful enough to realize all 'ancestor-simulations', and each and every simulated person in this Matrix-like virtual reality will have his/her consciousness embedded in those computers. In a nutshell, what Bostrom assumes for his argument is: (1) our future computers will be good enough to realize the simulated worlds we may wish to have, and (2) the simulated people in those worlds are fully conscious if we accept a particular theory in the philosophy of mind. ${ }^{4}$

Bostrom also suggests, rather playfully, that it may be possible for simulated civilizations to run their own ancestorsimulations on some sort of virtual machines (in the sense that they run computers in computers). This opens a possibility of ancestor-simulations creating hierarchically one-level lower ancestor simulations, ad infinitum. This logically infinitely simulation-creating computers may be 'prohibitively expensive' (let alone silly-sounding), so Bostrom again shrewdly suggests that our simulation would be 'terminated when we are about to become posthuman'. At any rate, what really lies behind his argument is that we cannot exclude the possibility of already living in a computer simulation.

Bostrom's argument naturally leads us to draw the conclusion that if the resources to run ancestor-simulations do not dry out, we probably will live almost forever in those simulations. This is Bostrom's way of attaining immortality through running computer simulations and our consciousness in them. However, the immortality attained this way leaves us a question: Can we call this immortal when we, as beings in a computer simulation, can be easily turned off pro re nata? Aren't we just puppets of our descendents who build computer simulations of us and our world? If we are to live in a computer

\footnotetext{
2 This has caused a lot of misunderstanding that Bostrom declares that we are living in a simulated reality. However, it is one possible environment in which we might end up in the very distant future. As far as the simulation argument itself is concerned, Bostrom's logic is consistent. For criticisms on Bostrom's argument and his replies to them, see Weatherson[13]-Bostrom[2] and Brueckner[4]-Bostrom[3] in pairs.

${ }^{3}$ Hans Moravec[8] also gives a very insightful estimation of how human intelligence can be realized in computers.

${ }^{4}$ See section 4 for discussions on human consciousness.
}

simulation, why this miserable world again $?^{5}$

\subsection{Kurzweil's Singularity}

Kurzweil is a radically optimistic futurist when it comes to the issue of artificial intelligence and our future life in an ultimate technological setting. One of the grounds for his future prospect on the issue comes from the exponential growth of computing that we have witnessed and will do in the near future. As shown in Fig. 2 taken from his book titled "The Singularity is near"[5], he expects that a $\$ 1,000$ personal computer will have as much as raw power than the human brain in 2019, and the same-priced personal computer will be 1,000 times more powerful than the human brain. In 2099, the human brain will have been completely reverse-engineered and all aspects of its functioning are understood. By this time, natural human thinking will possess no advantages over computer minds.

As he discusses immortality, Kurzweil covers a wide range of technological issues including conquering deadly diseases, increasing longevity, human cloning, and nanobot-driven virtual reality. Among them, his view and prospect on VR is the most relevant to the discussions in this paper. According to his estimation, when we eliminate 50 percent of medical problems, human life expectancy extends to over 150 years. At 90 percent, it grows to over 500 years, and at 99 percent, over 1,000 years. And eventually, we will live forever when move toward a non-biological existence that, say, resides in some sort of virtual reality.

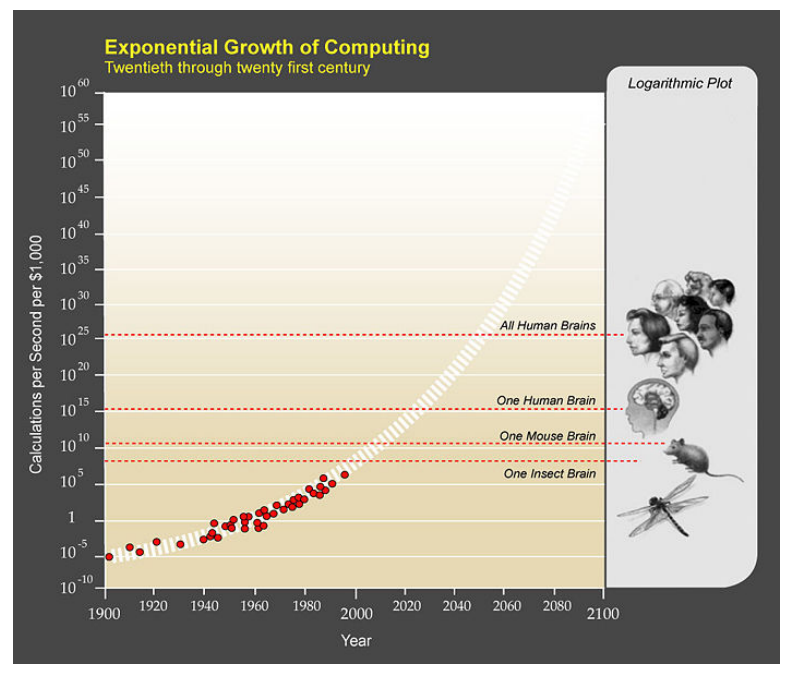

Fig. 2. Kurzweil's prospect on the exponential growth of computing in the logarithmic plot

Kurzweil's argument for immersive virtual reality and we in it is quite resonant with Bostrom's in that it predicts our technological advancement in an exponential phase will enable us to achieve virtual reality environments of the kind. Kurzweil's envisioning of our future is much more diverse and

\footnotetext{
5 It is not the purpose of this paper to criticize Bostrom's argument (and other arguments that follow), but to contemplate on the implications of technologically achieved immortality in the context of gaming environment.
} 
broad than that of Bostrom's. ${ }^{6}$ Kurzweil's VR is not limited to computer simulations (that is, software), but extended to nanobots in our brain which can toggle on and off our body, from real reality to virtual reality or the other way around. In his book, Kurzweil assertively says "In our brains, the massively distributed nanobots will interact with our biological neurons. This will provide full-immersion virtual reality incorporating all of the senses, as well as neurological correlates of our emotions, from within the nervous system."

One of the key ingredients in living in VR in the Kurzweil way is that we will exist as software-based humans in which all the information is represented in the patterns that we call our brains. That is, 'We' inside a computer simulation or VR eventually will be non-biological. Is this non-biological being based on my 'mind file' really me? This raises the questions of human consciousness and self-identity (see section 4), but if we accept the idea that our minds can be uploaded onto a computational system along with VR-projected bodies in it, we will, at least technologically, live, probably forever, in a VR environment.

\section{LIVING IN A COMPUTER GAME?}

Since their advent, we have been witnessing the advancement of computer games toward realizing more and more immersive and participatory user experience. Computer games developed by using very advanced game engines like Unreal Engine (Epic Games) boasts the not-so-very-differentfrom-reality graphic granularity. Considering the (measurably predictable) exponential growth of computer technology, it is not very difficult, as many futurists forecast, to expect that computer games (or computer simulation, if you like) in the not-so-distant future will be something indistinguishable from the world we live in.

In her book titled "Hamlet on the Holodeck"[8], regarded now as a classic work of its kind, Murray presents an imaginary example of how storytelling of dramas or games could be actualized in a futuristic holographic environment. In this imaginary interactive entertainment, the user named "Kathryn Janeway", captain of the starship Voyager, plays a role of the protagonist, creating new stories about her imaginary life, just like we continuously make new stories about our life as we live as a human being. However, it is not a virtual reality at the level of 'The Matrix' in that she turns off the program whenever she wants to come back to reality. It sure is an intermediate stage from which we go to the no-different-from-reality interactive environment (and possibly the don't-know-it's-avirtual-reality environment).

Probably the next step of progress in terms of gaming environment that we can imagine is the one in which it is no different from the real world, although we are still able to toggle between the virtual reality world and our real world. As suggested in Kurzweil[5], we may live two lives, i.e., the real

\footnotetext{
${ }^{6}$ Bostrom's main concern was not whether we will be living in a computer simulation, but rather the logical possibility of already living in it. Thus, it is not fair to say that Bostrom's vision of the future is near-sighted because his argument is not about how to implement a computer simulation or VR.
}

one and the virtual one. The Kurzweil kind of virtual reality would not look like the gaming environment that we know today, rather our body itself with nanobots called "foglets" inside of it would be a gaming environment, which we may take as the ultimate form of RPG (role-playing game). At this level of technological advancement, the distinction between gaming and living would be blurred. To express it in a more radical manner, gaming would be living. To be modest, gaming could be just one more life to live that nanotechnology and computer technology would offer to us.

Even with our current understanding of cosmology, it doesn't sound a Sci-Fi hoax any more that someday in the far distant future, we may have to migrate to an earth-like place by colonizing it. Considering the fate of the universe as we currently understands it, we may even have to give up our flesh and bones and exist as a software agent in a virtual reality. This is, though not exactly the same in every respect, what we are going to be in the ultimate future envisaged by renowned academics and futurists such as Kurzweil[5], Tipler[11, 12] and Moravec[8, 9]. Futuristic computer games would be our ultimate virtual world in which our conscious mind lives as some sort of software agent. In this future, computer games as VR systems and we in it are not a surplus world beneficially given to us, but it is our ultimate reality.

\section{CONSCIOUSNESS: AN UNSOLVED MYSTERY}

The issue of living in a computer simulation inevitably becomes intertwined with that of consciousness. We, as virtual beings without consciousness, would mean nothing at all. Fair enough. Let's face it, then! Am I really me in the system onto which my scanned brain (that is to say, the whole of my consciousness) has been uploaded? Human consciousness is one of the most difficult problems which the philosophy of mind has been struggling with. Though the issues and problems involving consciousness have been discussed more actively than ever before in various academic disciplines including philosophy, psychology, neuroscience, computer science, etc., it does not seem unfair to say we are still fumbling in the dark for the exact nature of human consciousness.

All of the three arguments in Section 2 for our living in some sort of VRs in the future with the whole of consciousness intact are based on the assumption that consciousness or conscious experiences can be somehow replicated. Though he admits it is controversial, Bostrom takes as his basic assumption on human consciousness the so-called 'Substrate-Independence Hypothesis' according to which consciousness can supervene on a variety of physical substrates. Kurzweil also discusses this issue at length mainly by criticizing Searle's Chinese Room Argument. Kurzweil sees human consciousness as some sort of emergent property which arises from complex patterns of brain states. So, he argues, rather simple-mindedly, that if we implement those brain states in whatever possible ways (in form of either computer software or nanobots), human consciousness naturally reveal itself. Human consciousness is one of the most difficult problems in the philosophy of mind, and we still have a long way to go before we know for sure whether or not it is reducible to the neurobiological states. If 
we don't solve the problem of consciousness, we still suffer from the problem of self-identity whatever systems we build for the residence of our consciousness. If we ever solve the mystery of consciousness, we will know for sure whether the ultimate VRs addressed in this paper will be our future adobe for human consciousness, with our yearning for living forever fulfilled.

\section{CONCLUDING REMARKS}

In this paper, we have discussed the issue of living (probably forever) in a far-advanced artificial environment from the perspective of computer engineering and related technologies. The two important arguments, Bostrom's Simulation Argument and Kurzweil's Singularity, for our ultimate VR-based adobe probably to be attained in the future have been introduced and reviewed. It was also discussed how this VR-based future technological setting relates to the future gaming environment. However, human consciousness still is a big problem to solve however we actualize VR systems that are not distinguishable from our real reality. Ironically, we will know for sure whether these ultimate VR systems with our yearning for immortality fulfilled when we fully understand the mystery of human consciousness.

\section{REFERENCES}

[1] Bostrom, Nick, "Are we living in a computer simulation?" Philosophical Quarterly, vol. 53, no. 211, 2003, pp. 211, 243-255.

[2] Bostrom, Nick, "The Simulation Argument: Reply to Weatherson", Philosophical Quarterly, vol. 55, no. 218, 2005, pp. 90-97.

[3] Bostrom, Nick, "The Simulation Argument: Some Explanations," Analysis, in press.

[4] Brueckner, A., "The Simulation Argument Again," Analysis, vol. 68, no. 3, 2008, pp. 224-226.

[5] Kurzweil, Ray, The Singularity Is Near, Penguin Books, New York, 2005.

[6] Lee, K. H., "As the story continues: The future of game storytelling," Journal of Korean Society of Computer Game, no. 23, 2010, pp. 277-282.

[7] Llinás, Rodolfo R., I of the Vortex, MIT Press, Massachusetts, 2002.

[8] Moravec, Hans, Mind Children: The Future of Robot and Human Intelligence, Harvard University Press, Cambridge, 1988.

[9] Moravec, Hans, Robot: Mere Machines to Transcendent Mind, Oxford University Press, Oxford, 1999.

[10] Murray, Janet H., Hamlet on the Holodeck: The Future of Narrative in Cyberspace, The Free Press, New York, 1997.

[11] Tipler, Frank J., The Physics of Immortality, Anchor Books, New York, 1995.

[12] Tipler Frank J., The Physics of Christianity, Doubleday, New York, 2007.

[13] Weatherson, Brian, "Are You a Sim?" Philosophical
Quarterly, vol. 53, 2003, pp. 425-31.

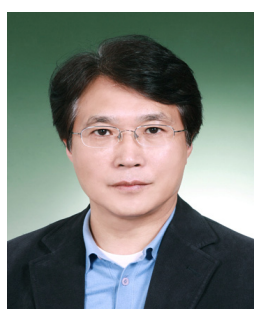

\section{Kang-Hyuk Lee}

He received his Ph.D. from University of Illinois at Urbana-Champaign, USA in 1993. During 1993-1996, he was with KAIST and KISTI as a postdoctoral research fellow and a senior research scientist, respectively. Since 1997, he has been teaching in the Dept. of Multimedia and Game Engineering at Tongmyong University. His current research interests include Game AI, Game Design, and Digital Storytelling. 